\title{
Class numbers of positive definite quinary quadratic forms
}

\author{
By Yoshiyuki KITAoKa
}

(Received December 17, 1974)

To know class numbers of positive definite quadratic forms with many variables there are three main tools, namely, the theory of adjancent lattices ([4], [6], [7]), Selberg trace formula ([8], [9], [10]), and Siegel formula ([2], [3], [5], [12]*). The first may be adapted for cases with small class numbers. The second was applied to quaternary forms with the theory of quaternions, but it seems to be difficult to apply this to other cases. Our aim is to show that the third is effective to the quinary forms, by proving

THEOREM. Let $p$ be a prime satisfying $p \equiv 1(8),\left(\frac{p}{5}\right)=1$; then the class number of quinary even**) positive definite quadratic lattices with discriminant $2 p$ is

$$
\begin{aligned}
& \frac{1}{2^{7} \cdot 3^{2} \cdot 5}\left(p^{2}-1\right)+\frac{7}{2^{6} \cdot 3} B_{2, x}+\frac{1}{144}\left(3+\left(\frac{p}{3}\right)\right)\left(p-\left(\frac{p}{3}\right)\right)+\frac{17}{2^{6} \cdot 3}(p-1) \\
& -\frac{1}{48}\left(3 p-5+2\left(\frac{p}{3}\right)\right)+\frac{1}{6} h(\sqrt{-3 p})+\frac{1}{16} h(\sqrt{-2 p})+\frac{1}{32} h(\sqrt{-p}),
\end{aligned}
$$

where $B_{2, x}$ is a generalized Bernoulli number with $\chi(n)=\left(\frac{n}{p}\right)$ and $h(\sqrt{-m})$ is the class number of quadratic field $Q(\sqrt{-m})$, and $(-)$ is Legendre symbol.

Notations and terminology will generally be those of O'Meara [7], and through this paper $Q(x)$ and $B(x, y)$ denote quadratic forms and corresponding bilinear forms (i.e., $2 B(x, y)=Q(x+y)-Q(x)-Q(y)$ ), respectively. We denote by $p$ a prime satisfying $p \equiv 1(8),\left(\frac{p}{5}\right)=1$, and $p$ is fixed through-
out the paper.

To get our theorem, we must classify lattices with non-trivial units. Hereafter $L$ stands for quinary even positive definite quadratic lattices with discriminant $2 p$.

*) Tsuge's result says: Let $p$ be prime; then $2 \times$ the class number of even positive definite quaternary forms with discriminant $\left.p^{2}\right\}-\{$ the class number of them in the narrow sense is the class number of the quaternion algebra $(p, \infty / Q)$.

**) "Even" means that the norm of quadratic lattice is divisible by 2 . 
0. $L$ is decomposable if and only if some vector $v \in L$ with $Q(v)=2$ splits $L$. Hence

$$
\sum_{L: \text { decomp. }}|O(L)|^{-1}=2^{-7} \cdot 3^{-1} B_{2, x},
$$

and the class number of decomposable lattices is

$$
2^{-5} \cdot 3^{-1} B_{2, \chi}+\frac{1}{48}\left(p+3-4\left(\frac{p}{3}\right)\right)+\frac{1}{12} h(\sqrt{-3 p})+\frac{1}{16} h(\sqrt{-p}),
$$

where $|O(L)|$ is the order of the group $O(L)$ of isometries of $L([3])$.

1. As a preliminary to classify indecomposable lattices we state the special cases of Th. 74.3 in [1].

Let $G=\langle\sigma\rangle$ be a cyclic group with prime order $q$; then we consider $X_{q}$ $=Z\left[1^{1 / q}\right] \oplus Z, Y_{q}=Z\left[1^{1 / q}\right]$ as $G$-modules by the following rules :

$$
\begin{array}{ll}
X_{q}: \sigma(w \oplus n)=\left(1^{1 / q} w \oplus n\right) \oplus n & \text { for } w \in Z\left[1^{1 / q}\right], n \in Z, \\
Y_{q}: \sigma w=1^{1 / q} w & \text { for } w \in Z\left[1^{1 / q}\right] .
\end{array}
$$

Moreover $Z_{a}$ stands for a $G$-trivial $Z$-module with rank $a$.

LEMma. Let $\sigma$ be a unit of $L$; then $L$ is isomorphic to one of the following modules as $\langle\sigma\rangle$-modules:

(i) $X_{5}$, (ii) $Y_{5} \oplus Z_{1}$ if the order of $\sigma$ is 5 ,

(i) $X_{3} \oplus Y_{3}$, (ii) $X_{3} \oplus Z_{2}$, (iii) $Y_{3} \oplus Y_{3} \oplus Z_{1}$, (iv) $Y_{3} \oplus Z_{3}$ if the order of $\sigma$ is 3 ,

(i) $X_{2} \oplus X_{2} \oplus Y_{2}$, (ii) $X_{2} \oplus X_{2} \oplus Z_{1}$, (iii) $X_{2} \oplus Y_{2} \oplus Y_{2} \oplus Y_{2}$, (iv) $X_{2} \oplus Y_{2} \oplus Y_{2} \oplus Z_{1}$, (v) $X_{2} \oplus Y_{2} \oplus Z_{2}$, (vi) $X_{2} \oplus Z_{3}$, (vii) $\oplus Y_{2} \oplus Z_{5-r}(0<r<5)$ if the order of $\sigma$ is 2 and $\sigma \neq-1$.

2. Lemma. If $L$ has a non-trivial unit, then $Q(L) \ni 2$.

Proof. If $L$ is decomposable, then $Q(L) \ni 2$ by $\mathbf{0}$. Hence we may assume that $L$ is indecomposable. If $L$ has a unit of order 5 , then $L$ is of type (i) since $L$ of type (ii) is decomposable. Therefore $L$ has a basis $\left\{e_{i}\right\}$ such that

$$
\left(B\left(e_{i}, e_{j}\right)\right)=\left(\begin{array}{ccccr}
2(a+b) & -b & -a & -a & -a-b \\
& 2(a+b) & -b & -a & -a \\
& & 2(a+b) & -b & 0 \\
& & & 2(a+b) & a \\
& & & & c
\end{array}\right),
$$

and $\operatorname{det}\left(B\left(e_{i}, e_{j}\right)\right)=\left(a^{2}+3 a b+b^{2}\right)^{2}(5 c-4 b-6 a)=2 p$. This implies $d Z\left[e_{1}, e_{2}\right.$, $\left.e_{3}, e_{4}\right]=5\left(a^{2}+3 a b+b^{2}\right)^{2}=5$. Hence $Q(L) \supset Q\left(Z\left[e_{1}, e_{2}, e_{3}, e_{4}\right]\right) \ni 2$. 
Assume that $L$ has a unit of order 3. If $L$ is of type (iii), (iv), then $L$ is decomposable. If $L$ is of type (i), then for some basis $\left\{e_{i}\right\}$ of $L$ we have

$$
\left(B\left(e_{i}, e_{j}\right)\right)=\left(\begin{array}{ccrcc}
2 a & -a & -a & -2 d-3 e & d \\
& 2 a & 0 & d+3 e & -2 d-3 e \\
& & b & d+e & e \\
* & & 2 c & -c \\
& & & & 2 c
\end{array}\right),
$$

and $\operatorname{det}\left(B\left(e_{i}, e_{j}\right)\right) \equiv 0(3)$. This is a contradiction. If $L$ is of type (ii), then for some basis $\left\{e_{i}\right\}$ of $L$ we have

$$
\left(B\left(e_{i}, e_{j}\right)\right)=\left(\begin{array}{rrrrr}
2 a & -a & -a & 0 & 0 \\
-a & 2 a & 0 & 0 & 0 \\
& & * & &
\end{array}\right.
$$

$2 p=\operatorname{det}\left(B\left(e_{i}, e_{j}\right)\right) \equiv 0\left(a^{2}\right)$. Hence $a=1$ and $Q(L) \ni 2$.

Unless $L$ has a unit of order 3 or $5,|O(L)|$ is a power of 2 . If, in this case, a unit of order 2 is only -1 , then there is some unit $\sigma$ of $L$ with $\sigma^{2}$ $=-1$. This is a contradiction since in this case $L$ is regarded as $Z[\sqrt{-1}]-$ torsion free module and rank $L$ is even. Hence we have a unit $\sigma(\neq-1)$ of order 2 unless $L$ has a unit of order 3 or 5 . If $L$ is of type (vii), then $L$ is decomposable. Since matrices of $\sigma$ corresponding to (i) and (ii), (iii) and (vi), or (iv) and (v), respectively, are conjugate in $G L(5, Z)$ up to \pm , we may assume that $L$ is of type (i), (vi) or (iv). If $L$ is of type (i), then

$$
\left(B\left(e_{i}, e_{j}\right)\right)=\left(\begin{array}{rrrrr}
a & b & c & d & g \\
& a & d & c & -g \\
& & e & f & h \\
* & & e & -h \\
& & & & l
\end{array}\right)
$$

for some basis $\left\{e_{i}\right\}$ of $L$. Put $N_{1}=Z\left[e_{1}+e_{2}, e_{3}+e_{4}\right], N_{2}=Z\left[e_{1}-e_{2}, e_{3}-e_{4}, e_{5}\right]$; then $N_{1}$ is orthogonal to $N_{2}$ and $\left[L: N_{1} \perp N_{2}\right]=4$, and from $d N_{1} \equiv 0(4), d N_{2}$ $\equiv 0$ (8) follows either $d N_{1}=4, d N_{2}=8 p$ or $d N_{1}=4 p, d N_{2}=8$. This implies $Q(L) \ni 2$ since $Q\left(N_{1}\right) \ni 2$ or $Q\left(N_{2}\right) \ni 2$ respectively. If $L$ is of type (vi), then

$$
\left(B\left(e_{i}, e_{j}\right)\right)=\left(\begin{array}{lllll}
a & b & c & d & e \\
b & a & c & d & e \\
& & * & &
\end{array}\right)
$$

for some basis $\left\{e_{i}\right\}$. Putting $v_{1}=e_{1}-e_{2}, v_{i}=e_{i}(i \geq 2)$, we have

$$
\left(B\left(v_{i}, v_{j}\right)\right)=\left(\begin{array}{ccccc}
2(a-b) & a-b & 0 & 0 & 0 \\
* & & &
\end{array}\right) .
$$


Hence $a-b \mid 2 p$ and $(a-b) d v_{1}^{\perp}=4 p$. Since $d v_{1}^{\perp} \equiv 0$ or 1(4), we have either $a-b=1, d v_{1}^{\perp}=4 p$ or $a-b=p, d v_{1}^{\perp}=4$. Hence $Q(L) \ni 2$. If $L$ is of type (iv), then

$$
\left(B\left(e_{i}, e_{j}\right)\right)=\left(\begin{array}{rrrrr}
a & b & c & d & e \\
& a & -c & -d & e \\
& & l & m & 0 \\
* & & n & 0 \\
& & & & t
\end{array}\right) .
$$

Put $N_{1}=Z\left[e_{1}+e_{2}, e_{5}\right], N_{2}=Z\left[e_{1}-e_{2}, e_{3}, e_{4}\right]$; then $N_{1}$ is orthogonal to $N_{2}$ and $\left[L: N_{1} \perp N_{2}\right]=2, d N_{1} \equiv 0(4), d N_{2} \equiv 0(2)$. Hence $d N_{1}=4$ and $Q(L) \supset Q\left(N_{1}\right) \ni 2$.

3. LEMMA 1. If $L$ is indecomposable and $Q(L) \ni 2$, then $L$ is written as $L(K)=Z\left[f_{2}\right] \perp K+Z\left[\left(f_{2}+u\right) / 2\right]$, where $Q\left(f_{2}\right)=2$, and $K$ satisfies

$$
d K=4 p, \quad K \cong\langle A(0,0)\rangle^{*)} \perp\langle-2\rangle \perp\langle 2\rangle \text { over } Z_{2},
$$

and $u \in K$ satisfies $Q(u) \equiv 6(8), B(u, K) \equiv 0(2)$, and then $u$ is uniquely determined modulo $2 K$ and $L(K)$ is uniquely determined by $K$. If, conversely, $K$ satisfies $(*)$, then $L(K)$ is even and $d L(K)=2 p$.

Proof. From our assumption there are a vector $f_{2} \in L$ with $Q\left(f_{2}\right)=2$, and a basis $\left\{f_{2}, e_{1}, e_{2}, e_{3}, e_{4}\right\}$ of $L$ such that $B\left(f_{2}, e_{1}\right)=1, B\left(f_{2}, e_{i}\right)=0(i \geq 2)$. Put $K=f_{2}^{\frac{1}{2}}=Z\left[f_{2}-2 e_{1}, e_{2}, e_{3}, e_{4}\right]$; then $d K=4 p, Q\left(f_{2}-2 e_{1}\right) \equiv 6(8), B\left(f_{2}-2 e_{1}, K\right)$ $\equiv 0(2)$. Hence $K \cong\langle A(2 a, 2 a)\rangle \perp\left\langle 2 \varepsilon_{1}\right\rangle \perp\left\langle 2 \varepsilon_{2}\right\rangle$ over $Z_{2}$ (a=0 or $1, \varepsilon_{i}$ : unit). 93 : 29 in [7] implies $K \cong\langle A(0,0)\rangle \perp\langle-2\rangle \perp\langle 2\rangle$ over $Z_{2}$. The rest is clear.

From here on $f_{2}$ stands for one used in Lemma 1.

LEMMA 2. If $L(K)$ contains a vector $y$ with $Q(y)=2$ except $\pm f_{2}$, then either $Q(K) \ni 2$ or $K$ has a vector $x$ satisfying $Q(x)=6, B(x, K) \equiv 0(2)$.

Proof. If $y=a\left(f_{2}+u\right) / 2+k \in L(K),(a \in Z, k \in K)$, is of $Q(y)=2$, then $Q(2 y)=2 a^{2}+Q(a u+2 k)=8$. If $a=0$, then $Q(K) \ni 2$. If $a= \pm 1$, then $x=a u$ $+2 k$ satisfies $Q(x)=6, B(x, K) \equiv 0(2)$. If $a= \pm 2$, then $y= \pm f_{2}$.

Hereafter we divide $K$ with $(*)$ to the following three types: $\equiv 0(2)$

(i) $Q(K) \not \supset 2$ and $K$ contains no vector $x$ satisfying $Q(x)=6, B(x, K)$

(ii) $K$ contains some vector $x$ satisfying $Q(x)=6, B(x, K) \equiv 0(2)$,

(iii) $Q(K) \ni 2$ and $K$ contains no vector $x$ satisfying $Q(x)=6, B(x, K)$ $\equiv 0(2)$.

\footnotetext{
*) $A(2 a, 2 a)$ stands for $\left(\begin{array}{cc}2 a & 1 \\ 1 & 2 a\end{array}\right)$.
} 
4. If $K$ is of type (i), then vectors of length 2 in $L(K)$ are $\pm f_{2}$, and $f_{2}$ does not split $L(K)$. Hence $L(K)$ is indecomposable, and $|O(L)|=2|O(K)|$. Moreover $L(K) \cong L\left(K_{1}\right)$ implies $K \cong K_{1}$ for any $K_{1}$ satisfying (*).

5. LEMMA 1. Let $K$ be of type (ii); then $K$ is constructed by $M$ as follows:

$$
K=K(M)=Z\left[f_{6}\right] \perp M+Z\left[\left(f_{6}+m\right) / 3\right],
$$

where $M$ satisfies

(**) $\quad M$ : even, $d M=6 p$, and $M \cong\langle 1\rangle \perp\langle 2 p\rangle \perp\langle 3\rangle$ over $Z_{3}$,

and $Q\left(f_{6}\right)=6$, and $m \in M$ satisfies $Q(m) \equiv 3(9), B(m, M) \equiv 0(3)$. Such a vector is unique up to \pm modulo $3 K$ and the isometry class of $K(M)$ is uniquely determined by $M$.

If, conversely, $M$ satisfies (**), then $K(M)$ is of type (ii).

Proof. From our assumption there is a vector $f_{6} \in K$ satisfying $Q\left(f_{6}\right)$ $=6, B\left(f_{6}, K\right) \equiv 0(2)$. Hence there is a basis $\left\{f_{6}, u_{1}, u_{2}, u_{3}\right\}$ of $K$ such that $B\left(f_{6}, u_{1}\right)=2, B\left(f_{6}, u_{i}\right)=0(i \geq 2)$. Put $M=f_{6}^{\frac{1}{6}}=Z\left[f_{6}-3 u_{1}, u_{2}, u_{3}\right]$; then $d M=6 p$, and $Q\left(f_{6}-3 u_{1}\right) \equiv 3(9), B\left(f_{6}-3 u_{1}, M\right) \equiv 0(3)$. Therefore $M \cong\langle 1\rangle \perp\langle 2 p\rangle \perp\langle 3\rangle$ over $Z_{3}$. The rest is clear.

In this case we put $L(M)=L(K(M))$; then we have $L(M)=Z\left[f_{2},\left(f_{2}+f_{6}\right) / 2\right.$, $\left.\left(f_{6}+m_{3}\right) / 3, m_{1}, m_{2}\right]$, where $\left\{m_{i}\right\}$ is a basis of $M$ such that

$$
\left(B\left(m_{i}, m_{j}\right)\right) \equiv\left(\begin{array}{lll}
1 & & \\
& 2 p & \\
& & 3
\end{array}\right) \bmod 9 .
$$

LEMMA 2. If $L(M)$ has a vector of length 2 except $\pm f_{2}, \pm\left(f_{2} \pm f_{6}\right) / 2$, then either $Q(M) \ni 2$ or $M$ has a vector $x$ such that $Q(x)=12$ and $B(x, M)$ $\equiv 0(3)$.

Proof. If $y=a f_{2}+b\left(f_{2}+f_{6}\right) / 2+c\left(f_{6}+m_{3}\right) / 3+d m_{1}+e m_{2} \in L(M)$ is of length 2 , then

$$
9(2 a+b)^{2}+3(3 b+2 c)^{2}+2 Q(x)=36,
$$

where $x=c m_{3}+3 d m_{1}+3 e m_{2}$.

If $2 a+b=3 b+2 c=0$, then $Q(M) \ni 2$. If either $2 a+b=0,3 b+2 c= \pm 2$ or $2 a+b= \pm 1,3 b+2 c= \pm 1$, then $Q(x)=12, B(x, M) \equiv 0(3)$. If $2 a+b= \pm 1$, $3 b+2 c= \pm 3$, then $y= \pm\left(f_{2} \pm f_{6}\right) / 2$. If $2 a+b= \pm 2$, then $y= \pm f_{2}$.

Accordingly we divide $M$ with (**) to the following three types: 
(a) $Q(M) \not 2$, and $M$ contains no vector $x$ such that $Q(x)=12, B(x, M)$ $\equiv 0(3)$,

(b) $Q(M) \ni 2$ and $M$ contains no vector $x$ such that $Q(x)=12, B(x, M)$ $\equiv 0(3)$,

(c) $M$ contains a vector $x$ such that $Q(x)=12, B(x, M) \equiv 0(3)$.

5.1. Lemma. If $M$ is of type (c), then $M$ is

$$
M(N)=Z\left[f_{12}\right] \perp N+Z\left[\left(f_{12}+n\right) / 4\right],
$$

where $N$ satisfies

$(* * *) \quad N:$ even, binary, $d N=8 p, S_{2}(N)^{*)}=-1$,

and $Q\left(f_{12}\right)=12$, and $n \in N$ satisfies $Q(n) \equiv 20(32), B(n, N) \equiv 0(4)$. The correspondence of $N$ and $M$ of type (c) is one to one for isometry classes, and $|O(M)|=|O(N)|$.

Proof. Let $M$ be of type (c); then there is $f_{12} \in M$ such that $Q\left(f_{12}\right)=12$, $B\left(f_{12}, M\right) \equiv 0(3)$. Hence we can take a basis $\left\{f_{12}, m_{1}, m_{2}\right\}$ of $M$ satisfying $B\left(f_{12}, m_{1}\right)=3, B\left(f_{12}, m_{2}\right)=0$. Put $N=f_{12}^{\frac{1}{2}}=Z\left[f_{12}-4 m_{1}, m_{2}\right]$; then $d N=8 p$, $S_{2}(N)=-1$, and $Q\left(f_{12}-4 m_{1}\right) \equiv 20(32), B\left(f_{12}-4 m_{1}, N\right) \equiv 0(4)$.

If, conversely, $N$ satisfies (***), then $N \cong\langle 2\rangle \perp\langle 4 \varepsilon\rangle$ over $Z_{2}$ where $\varepsilon=5$ or 7, moreover $n \in N$ satisfying $Q(n) \equiv 20(32), B(n, N) \equiv 0(4)$ exists uniquely up to \pm modulo $4 N$. Hence $M(N)$ is of type (c) and the isometry class of $M(N)$ is uniquely determined by $N$. To prove the rest it suffices to show that if $x \in M$ satisfies $Q(x)=12, B(x, M) \equiv 0(3)$, then $x= \pm f_{12}$. Take a basis $\left\{n_{j}\right\}$ of $N$ such that $Q\left(n_{1}\right) \equiv 20(32), B\left(n_{1}, N\right) \equiv 0(4)$, then we may put $M=$ $Z\left[\left(f_{12}+n_{1}\right) / 4, n_{1}, n_{2}\right]$. Assume that $x=a\left(f_{12}+n_{1}\right) / 4+b n_{1}+c n_{2} \in M$ satisfies $Q(x)=12, B(x, M) \equiv 0(3) . \quad B(x, M) \equiv 0(3)$ implies $a+4 b \equiv c \equiv 0(3)$. Hence we have

$$
4 a^{2}+3 Q\left(\frac{a+4 b}{3} n_{1}+\frac{4 c}{3} n_{2}\right)=64 .
$$

If $a= \pm 1$, then $y=[(a+4 b) / 3] n_{1}+(4 c / 3) n_{2}$ satisfies $Q(y)=20, B(y, N) \equiv 0(4)$. Hence $N \cong\left(\begin{array}{ll}20 & 4 a_{1} \\ 4 a_{1} & 2 a_{2}\end{array}\right)$ and $40 a_{2}-16 a_{1}^{2}=8 p$. This contradicts our assumption $\left(\frac{p}{5}\right)=1$. If $a= \pm 2$, then $Q(N) \ni 16$. This contradicts $S_{2}(N)=-1$. If $a=$ \pm 4 , then $x= \pm f_{12}$. Now the rest is clear.

5.2. Lemma. If $x \in K=K(M)$ of type (ii) satisfies $Q(x)=6, B(x, K)$ $\equiv 0(2)$ and $x \neq \pm f_{6}$, then $x= \pm \tau_{\left(f_{6}+f_{12}\right) / 3}\left(f_{6}\right)$ when $M$ is of type (c) and $K(M)$ $=Z\left[f_{6}\right] \perp M+Z\left[\left(f_{6}+f_{12}\right) / 3\right]$.

*) $S_{2}(N)$ is the Hasse symbol of $Q N$ at the prime two. 
Proof. Take a basis $\left\{m_{i}\right\}$ of $M$ such that

$$
\left(B\left(m_{i}, m_{j}\right)\right) \equiv\left(\begin{array}{lll}
1 & & \\
& 2 p & \\
& & 3
\end{array}\right) \bmod 9, \quad\left(B\left(m_{i}, m_{j}\right)\right) \equiv\left(\begin{array}{lll}
A(2 \delta, 2 \delta) & \\
& & 2
\end{array}\right) \bmod 8 .
$$

Suppose that $x=a f_{6}+b\left(f_{6}+m_{3}\right) / 3+c m_{1}+d m_{2} \in K(M)$ satisfies $Q(x)=6$, $B(x, K) \equiv 0(2) . \quad B(x, K) \equiv 0(2)$ implies $c \equiv d \equiv 0(2)$. Put $y=3 c m_{1}+3 d m_{2}+b m_{3}$; then $Q(y) \equiv 2 b^{2}(8)$, and $Q(3 x)=6(3 a+b)^{2}+Q(y)=54$. If $3 a+b= \pm 3$, then $x$ $= \pm f_{6}$. If $3 a+b= \pm 2$, then $Q(y)=30 \equiv 2 b^{2}(8)$ is a contradiction. If $3 a+b$ $= \pm 1$, then $Q(y)=48 \equiv 2 b^{2}(8)$ implies $b \equiv 0(2)$. Hence $\frac{1}{2} y \in M$ and $Q\left(\frac{1}{2} y\right)=12$, $B\left(\frac{1}{2} y, M\right) \equiv 0(3)$. From this follows that $M$ is of type (c). Hence $\frac{1}{2} y= \pm f_{12}$ in terminology of $\mathbf{5 . 1}$ and $3 x= \pm f_{6} \pm 2 f_{12} . x \in K(M)$ implies $x= \pm\left(\left(f_{6}+f_{12}\right) / 3\right.$ $\left.-f_{12}\right)= \pm \tau_{\left(f_{6}+f_{12}\right) / 3}\left(f_{6}\right)$. If $3 a+b=0$, then $Q(y)=54 \equiv 2 b^{2}(8)$ is a contradiction.

COROLlary. If $M$ is of type (a) or (b), then $|O(K)|=|O(M)|$. If $M$ is of type (c), then $|O(K)|=2|O(M)| . \quad K(M) \cong K\left(M_{1}\right)$ always implies $M \cong M_{1}$.

5.3. Lemma. Let $M$ be of type (a); then $L(M)$ is indecomposable and $|O(L)|=6|O(M)|$. If $L(M) \cong L\left(M_{1}\right)$, then $M \cong M_{1}$ for any $M_{1}$ satisfying $(* *)$.

Proof. The vectors of length 2 in $L(M)$ are $\pm f_{2}, \pm\left(f_{2} \pm f_{6}\right) / 2$ by Lemma 2, and any of them does not split $L(M)$. Hence $L(M)$ is indecomposable. Since a sublattice which is isometric to $\left(\begin{array}{ll}2 & 1 \\ 1 & 2\end{array}\right)$ is $Z\left[f_{2},\left(f_{2}+f_{6}\right) / 2\right]$, and $M=Z\left[f_{2},\left(f_{2}+f_{6}\right) / 2\right]^{\perp}$, the rest is clear.

5.4. Lemma. Let $M$ be of type (c); then $L(M)$ is indecomposable and $|O(L)|=24|O(M)|=24|O(N)| . \quad$ If $L(M(N)) \cong L\left(M\left(N_{1}\right)\right)$, then $N \cong N_{1}$.

Proof. In terminology of 5.1 we may assume $L(M(N))=Z\left[f_{2},\left(f_{2}+f_{6}\right) / 2\right.$, $\left.\left(f_{6}+f_{12}\right) / 3,\left(f_{12}+n_{1}\right) / 4, n_{2}\right]$ where $\left\{n_{i}\right\}$ is a basis of $N$ such that $Q\left(n_{1}\right) \equiv 20(32)$, $B\left(n_{1}, N\right) \equiv 0(4)$. Then the vectors of length 2 in $L(M)$ are $\pm f_{2}, \pm \tau_{\left(f_{2}-f_{6}\right) / 2}\left(f_{2}\right)$, $\pm \tau_{\left(f_{2}+f_{6}\right) / 2}\left(f_{2}\right), \quad \pm \tau_{f_{2}+\left(f_{6}+f_{12}\right) / 3-\left(f_{2}+f_{6}\right) / 2}\left(f_{2}\right), \quad \pm \tau_{\left(f_{6}+f_{12}\right) / 3-\left(f_{2}+f_{6}\right) / 2} \tau_{\left(f_{2}-f_{6}\right) / 2}\left(f_{2}\right)$, $\pm \tau_{\left(f_{6}+f_{12}\right) / 3-\left(f_{2}+f_{6}\right) / 2}\left(f_{2}\right)$, and these do not split $L(M)$. Hence $L(M)$ is indecomposable, and the sublattices of $L(M)$ which are isometric to $\left(\begin{array}{ll}2 & 1 \\ 1 & 2\end{array}\right)$ are $L_{0}$ $=Z\left[f_{2},\left(f_{2}-f_{6}\right) / 2\right], \tau_{f_{2}+\left(f_{6}+f_{12}\right) / 3-\left(f_{2}+f_{6}\right) / 2} L_{0}=Z\left[\left(f_{2}+f_{6}\right) / 2,\left(f_{6}+f_{12}\right) / 3\right], \tau_{\left(f_{6}+f_{12}\right) / 3}$

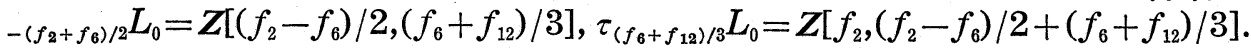
Now 5.1 and $M=L_{0}^{\perp}$ imply our lemma.

5.5. LEMma. Let $M$ be of type (b); then the only one of the following holds :

(1) $M=\langle 2\rangle \perp M_{1}$, where $M_{1}$ is even, $d M_{1}=3 p$, and $M_{1} \cong\langle p\rangle \perp\langle 3\rangle \mid Z_{3}$,

(2) $M=M(H)=Z\left[g_{2}\right] \perp H+Z\left[\left(g_{2}+h\right) / 2\right]$, where $H$ satisfies $d H=12 p, H$ 
$\cong\langle p\rangle \perp\langle 3\rangle / Z_{3}, H \cong\left\langle 2 \varepsilon_{1}\right\rangle \perp\left\langle 2 \varepsilon_{2}\right\rangle / Z_{2}\left(\varepsilon_{1}, \varepsilon_{2}:\right.$ units $)$, and $Q(H) \nexists 2$, and $h \in H$ satisfies $Q(h) \equiv 6(8)$.

Proof. From the assumption there is a vector $g_{2}$ in $M$ with $Q\left(g_{2}\right)=2$. If $g_{2}$ splits $M$, then $M$ is of type (1). If $M=\langle 2\rangle \perp M_{1}$ contains a vector $x$ such that $Q(x)=12, B(x, M) \equiv 0(3)$, then, putting $x=a g_{2}+m_{1}, m_{1} \in M_{1}$, we have $a \equiv 0(3), B\left(m_{1}, M_{1}\right) \equiv 0(3)$, especially $Q\left(m_{1}\right) \equiv 0(6)$. Now $Q(x)=2 a^{2}+Q\left(m_{1}\right)$ $=12$ implies $a=0$. Hence $M_{1} \cong\left(\begin{array}{ll}12 & 3 b \\ 3 b & 2 c\end{array}\right)$ with $24 c-9 b^{2}=3 p$. This contradicts $p \equiv 1(8)$. Assume that $g_{2}$ does not split $M$; then we can take a basis $\left\{g_{2}, v_{1}, v_{2}\right\}$ of $M$ such that $B\left(g_{2}, v_{1}\right)=1, B\left(g_{2}, v_{2}\right)=0$. Put $g_{\frac{1}{2}}=H=Z\left[g_{2}-2 v_{1}, v_{2}\right]$; then $d H=12 p, H \cong\langle p\rangle \perp\langle 3\rangle$ over $Z_{3}$ and $H \cong\left\langle 2 \varepsilon_{2}\right\rangle \perp\left\langle 2 \varepsilon_{1}\right\rangle$ over $Z_{2}$ since $Q\left(g_{2}-\right.$ $\left.2 v_{1}\right) \equiv 6(8)$. Now put $h_{1}=g_{2}-2 v_{1}, h_{2}=v_{2}$; then $M=M(H)=Z\left[g_{2},\left(g_{2}+h_{1}\right) / 2, h_{2}\right]$. If $x=a g_{2}+b\left(g_{2}+h_{1}\right) / 2+c h_{2} \in M(H)$ is of length $2, x= \pm g_{2}$ or $x \in H$. If $x \in H$, then $H \cong\left(\begin{array}{cc}2 & \\ & 6 p\end{array}\right)$ and $x$ splits $M(H)$. Hence in this case $M$ is of type (1). We must show that $M(H)$ does not contain such a vector $x$ that $Q(x)=12$, $B(x, M) \equiv 0(3)$. Assume that $x=a g_{2}+b\left(g_{2}+h_{1}\right) / 2+c h_{2}$ satisfies $Q(x)=12$, $B(x, M) \equiv 0(3)$; then $2 a+b \equiv b \equiv 0(3)$ and $Q(2 x)=2(2 a+b)^{2}+Q\left(b h_{1}+2 c h_{2}\right)=$ 48. If $2 a+b=0$, then $Q\left((b / 2) h_{1}+c h_{2}\right)=12$, and $B\left((b / 2) h_{1}+c h_{2}, H\right) \equiv 0(3)$. Hence we get $H \cong\left(\begin{array}{cc}12 & 6 \\ 6 & p+3\end{array}\right)$, but this contradicts $H \cong\left\langle 2 \varepsilon_{1}\right\rangle \perp\left\langle 2 \varepsilon_{2}\right\rangle$ over $Z_{2}$. If $2 a+b= \pm 3$, then $H \cong\left(\begin{array}{ll}30 & 6 a_{1} \\ 6 a_{1} & 2 a_{2}\end{array}\right)$ with $60 a_{2}-36 a_{1}^{2}=12 p$. This contradicts $\left(\frac{p}{5}\right)=1$.

COROLLARY. $M$ of type (b) is of type (2) if and only if $L(M)$ is indecomposable, and then the correspondence of $M$ and $H$ is bijective and $L(M) \cong$ $L\left(M_{1}\right)$ implies $M \cong M_{1}$ for any $M_{1}$ satisfying (**). Moreover $|O(L)|=6|O(M)|$ $=12|O(H)|$.

Proof. If $M$ is of type (1), then $L(M)$ is decomposable. Suppose that $M$ is of type (2); the vectors of length 2 in $L(M)$ are $\pm f_{2}, \pm\left(f_{2} \pm f_{6}\right) / 2, \pm g_{2}$, and these do not split $L(M)$. This implies that $L(M)$ is indecomposable. Since the sublattices of $L(M)$ which are isometric to $\left(\begin{array}{ll}2 & 1 \\ 1 & 2\end{array}\right)$ are $Z\left[f_{2},\left(f_{2}+f_{6}\right) / 2\right]$ and $M=Z\left[f_{2},\left(f_{2}+f_{6}\right) / 2\right]^{\perp}, M$ is uniquely determined by $L(M)$ and we have $|O(L)|=6|O(M)| . \quad h \in H$ satisfying $Q(h) \equiv 6(8)$ in Lemma is uniquely determined modulo $2 H$. Hence $M$ is uniquely determined by $H$. Since the vector of. length 2 in $M$ is unique up to \pm , the rest is clear.

5.6. We can calculate some quantities:

$$
\sum_{M: \text { type(c) }}|O(M)|^{-1}=\sum|O(N)|^{-1}=\frac{1}{8} h(\sqrt{-2 p}) .
$$




$$
\begin{aligned}
\sum_{\substack{M: L(M) \text { is } \\
\text { indecomposable }}}|O(M)|^{-1} & =\Sigma|O(M)|^{-1}-\frac{1}{2} \sum_{M_{1} \text { in } 5.5}\left|O\left(M_{1}\right)\right|^{-1} \\
& =\frac{1}{96}\left(3+\left(\frac{p}{3}\right)\right)\left(p-\left(\frac{p}{3}\right)\right)-\frac{1}{16} h(\sqrt{-3 p})
\end{aligned}
$$

Hence we have

$$
\begin{aligned}
& \sum_{L=L(K): \text { indecomposable }}|O(L)|^{-1}=\frac{1}{6} \sum_{M: \text { type(ii) }}|O(M)|^{-1} \\
& +\frac{1}{6} \sum_{\substack{M: t y p e(b) \\
\text { type }(2)}}|O(M)|^{-1}+\frac{1}{24} \sum_{M: t y p e(c)}|O(M)|^{-1} \\
& =\frac{1}{576}\left(3+\left(\frac{p}{3}\right)\right)\left(p-\left(\frac{p}{3}\right)\right) \\
& -\frac{1}{96} h(\sqrt{-3 p})-\frac{1}{64} h(\sqrt{-2 p}) \text {. } \\
& \sum_{K: \text { type (ii) }}|O(K)|^{-1}=\sum_{M: \operatorname{type}(a),(b)}|O(M)|^{-1}+\frac{1}{2} \sum_{M: \text { type (c) }}|O(M)|^{-1} \\
& =\frac{1}{96}\left(3+\left(\frac{p}{3}\right)\right)\left(p-\left(\frac{p}{3}\right)\right)-\frac{1}{16} h(\sqrt{-2 p}) .
\end{aligned}
$$

The class number of indecomposable $L(K)$, where $K$ is of type (ii), is

$$
\frac{1}{48}\left(3+\left(\frac{p}{3}\right)\right)\left(p-\left(\frac{p}{3}\right)\right)+\frac{1}{4} h(\sqrt{-3 p})-\frac{1}{8}\left(1-\left(\frac{p}{3}\right)\right)
$$

since the class number of $M$ is

$$
\frac{1}{48}\left(3+\left(\frac{p}{3}\right)\right)\left(p-\left(\frac{p}{3}\right)\right)+\frac{1}{2} h(\sqrt{-3 p})+\frac{1}{8}\left(1-\left(\frac{p}{3}\right)\right),
$$

and the class number of $M$ of type (b), (1) is

$$
\frac{1}{4} h(\sqrt{-3 p})+\frac{1}{4}\left(1-\left(\frac{p}{3}\right)\right) \text {. }
$$

The class number of $K$ of type (ii)

= The class number of $M$

$$
=\frac{1}{48}\left(3+\left(\frac{p}{3}\right)\right)\left(p-\left(\frac{p}{3}\right)\right)+\frac{1}{2} h(\sqrt{-3 p})+\frac{1}{8}\left(1-\left(\frac{p}{3}\right)\right) \text {. }
$$

6. LEMmA. A quadratic lattice $K$ is of type (iii) if and only if the only one of the following holds:

(1) $K=Z\left[g_{2}\right] \perp U$, where $Q\left(g_{2}\right)=2, d U=2 p, U \cong\langle A(0,0)\rangle \perp\langle-2\rangle$ over $Z_{2}$, and $U$ contains no vector $x$ such that $Q(x)=6, B(x, U) \equiv 0(2)$, 
(2) $K=Z\left[g_{2}\right] \perp T+Z\left[\left(g_{2}+t\right) / 2\right]$, where $T$ satisfies

$$
T: d T=8 p, T \cong\langle 2\rangle \perp\langle 6\rangle \perp\langle 6\rangle \text { over } Z_{2},
$$

and $Q(T) \nexists 2,4, Q(t) \equiv 6(8)$ and moreover $T$ contains no vector $x$ such that $Q(x)=6, B(x, t) \equiv 0(4)$.

Proof. There is a vector $g_{2}$ in $K$ with $Q\left(g_{2}\right)=2$ from our assumption. Firstly assume that $g_{2}$ splits $K$; then $K=Z\left[g_{2}\right] \perp U, d U=2 p, U \cong\langle A(0,0)\rangle \perp$ $\langle-2\rangle$ over $Z_{2}$. If $K$ has a vector $x=a g_{2}+u$, $(u \in U)$, satisfying $Q(x)=6$, $B(x, K) \equiv 0(2)$, then $Q(x)=2 a^{2}+Q(u)=6, B(u, U) \equiv 0(2)$. If $a=0$, then $Q(u)=6$. If $a= \pm 1$, then $Q(u)=4$. This is a contradiction. If, conversely, there is a vector $u \in U$ so that $Q(u)=6, B(u, U) \equiv 0(2)$, then $u$ satisfies $B(u, K) \equiv 0(2)$. Hence in this case the assertion (1) holds. Secondly assume that $g_{2}$ does not split $K$; then we can take a basis $\left\{g_{2}, w_{1}, w_{2}, w_{3}\right\}$ such that $B\left(g_{2}, w_{1}\right)=1, B\left(g_{2}, w_{i}\right)$ $=0,(i \geq 2)$. Put $T=g_{2}^{\perp}=Z\left[g_{2}-2 w_{1}, w_{2}, w_{3}\right]$; then $Q\left(g_{2}-2 w_{1}\right) \equiv 6(8), B\left(g_{2}-2 w_{1}\right.$, $T) \equiv 0(2), d T=8 p$, and $S_{2}(T)=-1$. From these follows $T \cong\langle 2\rangle \perp\langle 6\rangle \perp\langle 6\rangle$ over $Z_{2}$. Take a basis $\left\{t_{i}\right\}$ of $T$ such that $\left(B\left(t_{i}, t_{j}\right)\right) \equiv\left(\begin{array}{lll}2 & & \\ & 6 & \\ & & 6\end{array}\right) \bmod 8$; then $t \in T$ which satisfies $Q(t) \equiv 6(8)$ is $t_{1}+t_{2}+t_{3}, t_{2}$ or $t_{3}$ modulo $2 T$, and moreover $K$ constructed by $T$ as (2) in lemma satisfies (*) in Lemma 1 of 3 if and only if $t \equiv t_{2}$ or $t_{3} \bmod 2 T$. Hence $K=Z\left[g_{2}, t_{1}, t_{2},\left(g_{2}+t_{3}\right) / 2\right]$ or $Z\left[g_{2}, t_{1}, t_{3},\left(g_{2}+t_{2}\right) / 2\right]$ and $L(K)=Z\left[f_{2}, g_{2}, t_{1},\left(g_{2}+t_{3}\right) / 2,\left(f_{2}+t_{2}\right) / 2\right]$ or $Z\left[f_{2}, g_{2}, t_{1},\left(g_{2}+t_{2}\right) / 2,\left(f_{2}+t_{3}\right) / 2\right]$. Therefore the isometry class of $L(K)$ is uniquely determined by $T$. Assume that $K=Z\left[g_{2}, t_{1}, t_{2},\left(g_{2}+t_{3}\right) / 2\right]$ and $K$ contains a vector $y$ such that $Q(y)=6, B(y, K) \equiv 0(2)$; then $y=a g_{2}+\sum a_{i} t_{i}, a \equiv a_{3}(2) . \quad Q(y)=2 a^{2}$ $+Q\left(\sum a_{i} t_{i}\right)=6$. If $a=0$, then $Q\left(\sum a_{i} t_{i}\right)=6, B\left(\sum a_{i} t_{i}, t_{3}\right) \equiv 0(4)$. If $a= \pm 1$, then $Q\left(\sum a_{i} t_{i}\right)=4$. If, conversely, $T$ contains a vector $z$ of length 4 , then $Q\left(g_{2}+z\right)=6, B\left(g_{2}+z, K\right) \equiv 0(2)$ since $z \equiv t_{2}+t_{3} \bmod 2 T$. If $T$ contains a vector $z$ such that $Q(z)=6, B\left(z, t_{3}\right) \equiv 0(4)$, then $B(z, K) \equiv 0(2)$. Now we shall show that $Q(T) \ni 2$ if and only if there is a vector $x$ of length 2 such that $x$ splits $K$. The "only if" part is clear. If $x=a\left(g_{2}+t_{3}\right) / 2+\sum a_{i} t_{i}$ is of length 2 , then $Q(2 x)=2 a^{2}+Q\left(a t_{3}+2 \sum a_{i} t_{i}\right)=8$. If $a=0$, then $Q(T) \ni 2$, and $K$ is of type (1). If $a= \pm 1$, then $Q\left(x-a g_{2}\right)=2$ and $\tau_{x-a g_{2}}\left(a g_{2}\right)=x$. Hence $x$ does not split $K$. If $a= \pm 2$, then $x= \pm g_{2}$. This completes the proof.

CoROLlaRY. If $K$ is of type (2), then the isometry class of $L(K)$ is uniquely determined by $T$.

COROLlaRY. $\sum_{K: \text { type }(1)}|O(K)|^{-1}=\frac{1}{96}(p-1)-\frac{1}{16} h(\sqrt{-3 p})$,

and the class number of $K$ of type (1) is 


$$
\frac{1}{24}\left(p-3+2\left(\frac{p}{3}\right)\right)-\frac{1}{4} h(\sqrt{-3 p})+\frac{1}{4} h(\sqrt{-p}) .
$$

Proof. Let $U$ be a quadratic lattice such that $d U=2 p, U \cong\langle A(0,0)\rangle$ $\perp\langle-2\rangle$ over $Z_{2}$. If $U$ has a vector $g_{6}$ which satisfies $Q\left(g_{6}\right)=6, B\left(g_{6}, U\right) \equiv 0(2)$, then we can take a basis $\left\{g_{6}, u_{1}, u_{2}\right\}$ of $U$ such that $B\left(g_{6}, u_{1}\right)=2, B\left(g_{6}, u_{2}\right)=0$. Put $U_{1}=g_{6} \frac{1}{6}=Z\left[g_{6}-3 u_{1}, u_{2}\right]$; then $d U_{1}=3 p, U_{1} \cong\langle p\rangle \perp\langle 3\rangle$ over $Z_{3}$ and $U_{1}$ is even. Clearly the correspondence of $U$ and $U_{1}$ is bijective and $|O(U)|$ $=\left|O\left(U_{1}\right)\right|$. Hence

$$
\sum_{K: \text { type(1) }}|O(K)|^{-1}=\frac{1}{2}\left(\sum|O(U)|^{-1}-\sum\left|O\left(U_{1}\right)\right|^{-1}\right),
$$

and

$$
\sum|O(U)|^{-1}=\frac{1}{48}(p-1), \quad \sum\left|O\left(U_{1}\right)\right|^{-1}=\frac{1}{8} h(\sqrt{-3 p})
$$

imply the first equation. The class number of $U$ which satisfies $U \cong$ $\langle A(0,0)\rangle \perp\langle-2\rangle$ over $Z_{2}, d U=2 p$ is $\left(p+3-4\left(\frac{p}{3}\right)\right) / 24+h(\sqrt{-p}) / 4$ and the class number of $U_{1}$ is $h(\sqrt{-3 p}) / 4+\left(1-\left(\frac{p}{3}\right)\right) / 4$. This completes the proof.

6.1. Lemma. A quadratic lattice $T$ which satisfies (*) and $Q(T) \nexists 2,4$, $Q(T) \ni 6$ is written as $T=Z\left[g_{6}\right] \perp V+Z\left[\left(g_{6}+v\right) / 3\right]$, where $Q\left(g_{6}\right)=6, d V=12 p$, $V \cong\langle p\rangle \perp\langle 3\rangle$ over $Z_{3}, V \cong\langle 2\rangle \perp\langle 6\rangle$ over $Z_{2}$, and $Q(V) \nexists 2$, and moreover $v \in V$ satisfies $Q(v) \equiv 3(9), B(v, V) \equiv 0(3)$. Then the correspondence of $T$ and $V$ is bijective, and $|O(T)|=|O(V)|$.

Proof. We can take a basis $\left\{g_{6}, z_{1}, z_{2}\right\}$ of $T$ such that $Q\left(g_{6}\right)=6, B\left(g_{6}, z_{1}\right)$ $=2, B\left(g_{6}, z_{2}\right)=0$. Put $V=g_{6}^{\frac{1}{6}}=Z\left[g_{6}-3 z_{1}, z_{2}\right]$; then $Q\left(g_{6}-3 z_{1}\right) \equiv 3(9), B\left(g_{6}-3 z_{1}\right.$, $V) \equiv 0(3), d V=12 p$. These imply $V \cong\langle p\rangle \perp\langle 3\rangle$ over $Z_{3}, V \cong\langle 2\rangle \perp\langle 6\rangle$ over $Z_{2}$. Conversely for such a $V$, we take a basis $\left\{v_{i}\right\}$ of $V$ such that

$$
\left(B\left(v_{i}, v_{j}\right)\right) \equiv\left(\begin{array}{ll}
3 & \\
& p
\end{array}\right)(9) \text { and }\left(B\left(v_{i}, v_{j}\right)\right) \equiv\left(\begin{array}{ll}
2 & \\
& 6
\end{array}\right)(8)
$$

If, now, $Q(v) \equiv 3(9), B(v, V) \equiv 0(3)$, then $v \equiv \pm v_{1} \bmod 3 V$. We shall show $Q(T) \nexists 2,4$ for $T$ as constructed in Lemma. If $Q(T) \ni 2$, then putting $x=$ $a\left(g_{6}+v_{1}\right) / 3+b v_{1}+c v_{2} \in T, Q(x)=2$ implies $6 a^{2}+Q\left(a v_{1}+3\left(b v_{1}+c v_{2}\right)\right)=18$. If $a=0$, then $Q(V) \ni 2$. This is a contradiction. If $a= \pm 1$, then $Q(V) \ni 12$ and $V \cong\left(\begin{array}{cc}12 & 6 \\ 6 & p+3\end{array}\right)$. This contradicts $V \cong\langle 2\rangle \perp\langle 6\rangle$ over $Z_{2}$. Hence $Q(T)$ $\nexists 2$. If $Q(x)=4$, then $6 a^{2}+Q\left(a v_{1}+3\left(b v_{1}+c v_{2}\right)\right)=36$. If $a=0$, then $Q(V) \ni 4$. 
This contradicts $S_{2}(V)=1$. If $a= \pm 1$, then $Q(V) \ni 30$ and $V \cong\left(\begin{array}{ll}30 & 6 x \\ 6 x & 2 y\end{array}\right)$, $60 y-36 x^{2}=12 p$. This contradicts $\left(\frac{p}{5}\right)=1$. If $a= \pm 2$, then $Q(V) \ni 12$.

Hence $Q(V) \nexists 4$. To prove the rest it suffices to show that a vector in $T$ of length 6 is $\pm g_{6}$. Let $x=a\left(g_{6}+v_{1}\right) / 3+b v_{1}+c v_{2} \in T$ be of length 6 ; then $Q(3 x)$ $=6 a^{2}+Q\left(a v_{1}+3\left(b v_{1}+c v_{2}\right)\right)=54$. If $a=0$, then $Q(V) \ni 6$. This contradicts $V \cong\langle p\rangle \perp\langle 3\rangle$ over $Z_{3}$. If $a= \pm 1$, then $V$ contains a vector $y$ such that $Q(y)$ $=48, B(y, V) \equiv 0(3)$. If $y$ is maximal, then $V \cong\left(\begin{array}{ll}48 & 6 x \\ 6 x & 2 y\end{array}\right), 96 y-36 x^{2}=12 p$. This contradicts $p \equiv 1(8)$. If $y$ is not maximal, then $V \cong\left(\begin{array}{cc}12 & 6 \\ 6 & p+3\end{array}\right)$. This is also a contradiction. If $a= \pm 2$, then $Q(V) \ni 30$, which contradicts $\left(\frac{p}{5}\right)$ $=1$. If $a= \pm 3$, then $x= \pm g_{6}$. This completes our proof.

COROLLARY.

$$
\sum_{\substack{Q(T, P 2,4 \\ Q(T) \geqslant B}}|O(T)|^{-1}=\frac{3}{8} h(\sqrt{-3 p})-\frac{1}{8}\left(1-\left(\frac{p}{3}\right)\right),
$$

and the class number of $T$ with $Q(T) \nexists 2,4, Q(T) \ni 6$ is

$$
\frac{3}{4} h(\sqrt{-3 p})-\frac{1}{4}\left(1-\left(\frac{p}{3}\right)\right) \text {. }
$$

CoROLlaRY. Let $T$ satisfy $(\stackrel{*}{*}), Q(T) \not \supset 2,4$, and $Q(T) \ni 6$; then there is a unique vector $t$ modulo $2 T$ such that $K=Z\left[g_{2}\right] \perp T+Z\left[\left(g_{2}+t\right) / 2\right]$ is of type (iii), and then $|O(K)|=6|O(V)|$.

Proof. Put $t_{1}=g_{6}+\left(g_{6}+v_{1}\right) / 3, t_{2}=v_{2}, t_{3}=g_{6}$, where $\left\{v_{i}\right\}$ is a basis of $V$ such that $\left(B\left(v_{i}, v_{j}\right)\right) \equiv\left(\begin{array}{ll}3 & \\ & p\end{array}\right)(9),\left(B\left(v_{i}, v_{j}\right)\right) \equiv\left(\begin{array}{ll}2 & \\ & 6\end{array}\right)(8)$; then $\left(B\left(t_{i}, t_{j}\right)\right) \equiv$ $\left(\begin{array}{lll}2 & & \\ & 6 & \\ & & 6\end{array}\right)(8)$. Hence $t \equiv g_{6} \bmod 2 T$ if and only if $K$ is of type (iii).

6.2. Lemma. Let $K$ be of type (iii); then $L(K)$ is indecomposable and is not constructed by $K^{\prime}$ of type (ii) if and only if $K$ is of type (2) in Lemma of 6 and $Q(T) \nexists 2,4,6$. Then $|O(L(K))|=4|O(T)|$ and the correspondence of $L$ and $T$ is bijective.

Proof. If $K$ is of type (1), then $L(K)$ is decomposable. Let $K$ be of type (2); then we may assume that $L(K)=Z\left[\left(f_{2}+t_{2}\right) / 2,\left(g_{2}+t_{3}\right) / 2, t_{1}, t_{2}, t_{3}\right]$, where $\left\{t_{i}\right\}$ is a basis of $T$ so that $\left(B\left(t_{i}, t_{j}\right)\right) \equiv\left(\begin{array}{lll}2 & & \\ & 6 & \\ & & 6\end{array}\right)(8)$. Since the vectors of length 2 in $L(K)$ is $\pm f_{2}, \pm g_{2}$ if $Q(T) \nexists 2,4,6, L(K)$ is indecomposable and 
the sublattice in $L(K)$ which is isometric to $\left(\begin{array}{ll}2 & \\ & 2\end{array}\right)$ is only $Z\left[f_{2}, g_{2}\right]$. This implies the latter half. Assume that $L(K) \cong L(K(M))$, where $M$ satisfies (**) in 5. Let $\sigma$ be an isometry from $L(K)$ to $L(K(M))$. If $\sigma\left(f_{2}\right)$ is mapped to $f_{2}$ by $O(L(K(M))$ ), then $K \cong K(M)$. This is a contradiction. Hence we may suppose that $M$ is of type (b), (2) in $\mathbf{5 . 5}$ and $\sigma\left(f_{2}\right)=g_{2}$. Hence $K \cong g \frac{\perp}{2}=Z\left[f_{2}\right.$, $\left.\left(f_{2}+f_{6}\right) / 2,\left(f_{6}+h_{2}\right) / 2, h_{1}\right]$, where $\left\{h_{i}\right\}$ is a basis of $H$ such that $\left(B\left(h_{i}, h_{j}\right)\right) \equiv$

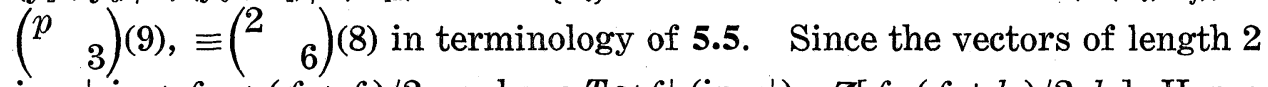
in $g_{2}^{\perp}$ is $\pm f_{2}, \pm\left(f_{2} \pm f_{6}\right) / 2$, we have $T \cong f_{2}^{\perp}$ (in $\left.g_{2}^{\perp}\right)=Z\left[f_{6},\left(f_{6}+h_{2}\right) / 2, h_{1}\right]$. Hence $Q(T) \ni 6$. Conversely suppose $Q(T) \ni 6$; then $T=Z\left[g_{6}\right] \perp V+Z\left[\left(g_{6}+v_{2}\right) / 3\right]$ as 6.1, where $\left\{v_{j}\right\}$ is a basis of $V$ such that $\left(B\left(v_{i}, v_{j}\right)\right) \equiv\left(\begin{array}{ll}p & 3\end{array}\right)(9), \equiv\left(\begin{array}{ll}2 & 6\end{array}\right)(8)$, and $L(K)=Z\left[\left(f_{2}+v_{2}\right) / 2,\left(g_{6}+v_{2}\right) / 3,\left(g_{2}+g_{6}\right) / 2, v_{1}, v_{2}\right]$. This $V$ is regarded as $H$ in 5.5. Put $M(V)=Z\left[f_{2}\right] \perp V+Z\left[\left(f_{2}+v_{2}\right) / 2\right], K(M)=Z\left[g_{6}\right] \perp M(V)+Z\left[\left(g_{6}\right.\right.$ $\left.\left.+v_{2}\right) / 3\right]$; then $K(M)$ is of type (ii), and $L(K)=Z\left[g_{2}\right] \perp K(M)+Z\left[\left(g_{2}+g_{6}\right) / 2\right]$. Hence $L(K)$ is constructed by $K^{\prime}$ of type (ii) if $Q(T) \ni 6$.

6.3. LEMma. If $T$ satisfies (*) and $Q(T) \ni 4$, then $T=Z\left[f_{4}\right] \perp W+$ $Z\left[\left(f_{4}+w\right) / 2\right]$, where $W \cong\langle 10\rangle \perp\langle 20\rangle$ over $Z_{2}, d W=8 p$ and $w \in W$ satisfies $Q(w) \equiv 4(8), B(w, W) \equiv 0(4)$; the correspondence of $T$ and $W$ is bijective, and $|O(T)|=2|O(W)|, Q(T) \nexists 2,6$.

Proof. From our assumption we can take a basis $\left\{f_{4}, t_{1}, t_{2}\right\}$ of $T$ such that $Q\left(f_{4}\right)=4, B\left(f_{4}, t_{1}\right)=2, B\left(f_{4}, t_{2}\right)=0$. Put $W=f_{4}^{1}=Z\left[f_{2}-2 t_{1}, t_{2}\right]$, then $d W=8 p$, $Q\left(f_{4}-2 t_{1}\right) \equiv 4(8), \quad B\left(f_{4}-2 t_{1}, W\right) \equiv 0(4) . \quad S_{2}(W)=-1$ implies $W \cong\langle 10\rangle \perp\langle 20\rangle$ over $\boldsymbol{Z}_{2}$, and $w \in W$ which satisfies $Q(w) \equiv 4(8), B(w, W) \equiv 0(4)$ is unique modulo $2 W$. From this follows that $T$ is uniquely determined by $W$. Since the vectors of length 4 in $T$ are $\pm f_{4}$, the correspondence of $T$ and $W$ is bijective and $|O(T)|=2|O(W)| . \quad Q(T) \nexists 2,6$ is easy to see.

CoRollaRY. $\quad \sum_{Q(T) \ni 4}|O(T)|^{-1}=(1 / 16) h(\sqrt{-2 p})$ and the class number of $T$ with $Q(T) \ni 4$ is $(1 / 4) h(\sqrt{-2 p})$.

6.4. Lemma. In (2) of Lemma in 6, $K$ is constructed uniquely by $T$ if $Q(T) \ni 6$, and if $Q(T) \nexists 6$, then there are two $K$ constructed by $T$, and they are not isometric.

Proof. By 6 and 6.1, we have only to prove the last part. If they are isometric, then there exists a unit $\sigma$ of $T$ such that $\sigma\left(t_{2}\right) \equiv t_{3}(2 T)$, where $\left\{t_{i}\right\}$ is a basis of $T$ satisfying $\left(B\left(t_{i}, t_{j}\right)\right) \equiv\left(\begin{array}{lll}2 & & \\ & 6 & \\ & & 6\end{array}\right)(8)$. If, however, $T$ has a nontrivial unit, then $Q(T) \ni 2$ or 4 . This completes our proof. 
Corollary. If $Q(T) \nexists 2,4,6$, then $|O(K)|=2|O(T)|$.

Proof. If $Q(T) \nexists 2,4$, then any unit $\sigma$ of $T$ satisfies $\sigma\left(t_{2}\right) \equiv t_{2} \bmod 2 T$. From this and 6 follows our Corollary.

6.5. LEMMA. $\quad \sum_{Q(T) \ni 2}|O(T)|^{-1}=(1 / 16) h(\sqrt{-p})$ and the class number of $T$ with $Q(T) \ni 2$ is $(1 / 4) h(\sqrt{-p})$.

Proof. If $Q(T) \ni 2$, then $T=\langle 2\rangle \perp T_{1}$, where $d T_{1}=4 p, T \cong\langle 6\rangle \perp\langle 6\rangle \mid Z_{2}$. Now Lemma is clear.

To get the class number formula of $L$ we need a few more formulas.

An easy calculation shows

LEMMA.

$$
\sum_{T:(*)}|O(T)|^{-1}=\frac{1}{32}(p-1),
$$

and the class number of $T$ satisfying $(\underset{*}{*})$ is

$$
\frac{1}{16}(p-1)+\frac{1}{8} h(\sqrt{-2 p})+\frac{1}{8} h(\sqrt{-p}) .
$$

$\sum_{K:(*)}|O(K)|^{-1}=(1 / 32) B_{2, x}$, and the class number of $K$ satisfying $(*)$ is

$$
\frac{1}{16} B_{2, x}+\frac{1}{4} h(\sqrt{-3 p})+\frac{1}{8} h(\sqrt{-p})+\frac{1}{12}\left(p-\left(\frac{p}{3}\right)\right),
$$

and lastly $\sum|O(L)|^{-1}=\left(p^{2}-1\right) /\left(2^{8} \cdot 3^{2} \cdot 5\right)$.

7. Under all the above preliminalies we can calculate the class number of $L$ :

$$
\begin{aligned}
\sum|O(L)|^{-1}= & \frac{1}{4} \sum_{Q(T) \nexists 2,4,6}|O(T)|^{-1} \\
= & \frac{1}{2^{7}}(p-1)+\frac{1}{32}\left(1-\left(\frac{p}{3}\right)\right)-\frac{3}{32} h(\sqrt{-3 p}) \\
& -\frac{1}{64} h(\sqrt{-2 p})-\frac{1}{64} h(\sqrt{-p}),
\end{aligned}
$$

where $L$ runs over the indecomposable quadratic lattices which are constructed by $K$ of (iii), but not of type (ii).

The class number of $L$ such that $L$ is indecomposable and is constructed by $K$ of type (iii), but not of type (ii)

$$
\begin{aligned}
& =\text { the class number of } T \text { with } Q(T) \nexists 2,4,6 \\
& =\frac{1}{16}(p-1)+\frac{1}{4}\left(1-\left(\frac{p}{3}\right)\right)-\frac{3}{4} h(\sqrt{-3 p})-\frac{1}{8} h(\sqrt{-2 p})-\frac{1}{8} h(\sqrt{-p}) .
\end{aligned}
$$




$$
\begin{aligned}
\sum_{K: \text { type (i) }}|O(L(K))|^{-1}= & \frac{1}{2} \sum_{K: \text { type (i) }}|O(K)|^{-1} \\
= & \frac{1}{2}\left(\sum|O(K)|^{-1}-\sum_{K: \text { type (ii), (iii) }}|O(K)|^{-1}\right) \\
= & \frac{1}{64} B_{2, x}-\frac{1}{192}\left(3+\left(\frac{p}{3}\right)\right)\left(p-\left(\frac{p}{3}\right)\right)+\frac{3}{16} h(\sqrt{-3 p}) \\
& +\frac{1}{16} h(\sqrt{-2 p})+\frac{1}{32} h(\sqrt{-p})-\frac{1}{48}(p-1) \\
& -\frac{5}{96}\left(1-\left(\frac{p}{3}\right)\right) .
\end{aligned}
$$

The class number of $K$ of type (i)

$$
\begin{aligned}
= & \frac{1}{16} B_{2, x}-\frac{1}{48}\left(3+\left(\frac{p}{3}\right)\right)\left(p-\left(\frac{p}{3}\right)\right)-\frac{1}{24}\left(2 p+3-5\left(\frac{p}{3}\right)\right) \\
& +\frac{3}{4} h(\sqrt{-3 p})+\frac{1}{4} h(\sqrt{-2 p})+\frac{1}{8} h(\sqrt{-p}) .
\end{aligned}
$$

Hence the class number of $L=2\left(\sum|O(L)|^{-1}-\sum_{|O(L)|>2}|O(L)|^{-1}\right)+\{$ the class number of $K$ of type (i) $\}+\{$ the class number of $K$ of type (ii) such that $L(K)$ is indecomposable $\}+\{$ the class number of $K$ of type (iii) such that $L(K)$ is indecomposable and is not constructed by $K^{\prime}$ of type (ii) $\}+\{$ the class number of decomposable $L\}$

$$
\begin{aligned}
= & \frac{1}{2^{7} \cdot 3^{2} \cdot 5}\left(p^{2}-1\right)+\frac{7}{2^{6} \cdot 3} B_{2, x}+\frac{1}{144}\left(3+\left(\frac{p}{3}\right)\right)\left(p-\left(\frac{p}{3}\right)\right)+\frac{17}{2^{6} \cdot 3}(p-1) \\
& -\frac{1}{48}\left(3 p-5+2\left(\frac{p}{3}\right)\right)+\frac{1}{6} h(\sqrt{-3 p})+\frac{1}{16} h(\sqrt{-2 p})+\frac{1}{32} h(\sqrt{-p}) .
\end{aligned}
$$

\section{References}

[1] C. W. Curtis and I. Reiner, Representation theory of finite groups and associative algebras, Interscience Pub., 1962.

[2] G. Eisenstein, Tabelle der reducirten positiven ternären quadratischen Formen, J. für Math., 41 (1851), 141-190.

[3] Y. Kitaoka, Quaternary even positive definite quadratic forms of prime discriminant, Nagoya Math. J., 52 (1973), 147-161.

[ 4 ] M. Kneser, Klassenzahlen definiter quadratischer Formen, Arch. Math., 8 (1957), 241-250.

[5] L. J. Mordell, On the class number for definite ternary quadratics, Messenger of Math., 47 (1918), 65-78.

[6] H. V. Niemeier, Definite quadratische Formen der Dimension 24 und Diskriminante 1, J. Number Theory, 5 (1973), 142-178. 
[ 7 ] O. T. O'Meara, Introduction to quadratic forms, Springer-Verlag, 1963.

[ 8 ] P. Ponomarev, Class numbers of positive definite quaternary forms, Bull. Amer. Math. Soc., 76 (1970), 646-649.

[9] - Class number of definite quaternary forms with nonsquare discriminant, Bull. Amer. Math. Soc., 79 (1973), 594-598.

[10] _ Class numbers of definite quaternary forms with square discriminant, J. Number Theory, 6 (1974), 291-317.

[11] C. L. Siegel, Über die analytische Theorie der quadratischen Formen, Ann. of Math., 36 (1935), 527-606.

[12] T. Tsuge, On class numbers of definite quaternary forms, (unpublished).

DEPARTMENT OF MATHEMatics

NAgOYA UNIVERSITY 